\title{
Comparison of several eigenvalue reanalysis methods for modified structures
}

by Chen SH, Yang XW, Lian HD

Struct Multidisc Optim 20(4), 253-259 (2000)

In the above review paper, the authors used repeatedly the terms "Pade' approximate method" and "Pade' approximation". On the basis of the literature on this topic,
"Pade" should read "Padé" in these expressions. The Editor wishes to thank Prof. R.T. Haftka for advising him on this issue. 